\title{
Compreendendo a influência do fotoperíodo no cultivo de Litopenaeus vannamei: revisitando estudos realizados para o período de 2005-2020
}

\author{
Understanding the influence of photoperiod on the cultivation of Litopenaeus vannamei: revisiting
} studies carried out for the $2005-2020$ cropping period

Comprensión de la influencia del fotoperíodo en el cultivo de Litopenaeus vannamei: revisión de los estudios realizados para el período de cultivo 2005-2020

Recebido: 24/07/2021 | Revisado: 01/08/2021 | Aceito: 10/08/2021 | Publicado: 14/08/2021

\author{
Denise de Barros Nogueira \\ ORCID: https://orcid.org/0000-0002-1256-1062 \\ Universidade Federal Rural de Pernambuco, Brasil \\ E-mail: denisebarros1993@gmail.com \\ Tays Ferreira Barros \\ ORCID: https://orcid.org/0000-0002-2807-4488 \\ Universidade Federal Rural de Pernambuco, Brasil \\ E-mail: tayssssferreira@live.com \\ Luciana Sandra Bastos de Souza \\ ORCID: https://orcid.org/0000-0001-8870-0295 \\ Universidade Federal Rural de Pernambuco, Brasil \\ E-mail: sanddrabastos@yahoo.com.br \\ Wilma Roberta dos Santos \\ ORCID: https://orcid.org/0000-0001-6680-7884 \\ Universidade Federal Rural de Pernambuco, Brasil \\ E-mail: wilmaroberta1@gmail.com \\ George do Nascimento Araújo Júnior \\ ORCID: https://orcid.org/0000-0001-9284-4160 \\ Universidade Federal Rural de Pernambuco, Brasil \\ E-mail: georgearaujo.agro@gmail.com \\ Carlos André Alves de Souza \\ ORCID: https://orcid.org/0000-0003-1946-0421 \\ Universidade Federal Rural de Pernambuco, Brasil \\ E-mail: carlosandre08_@msn.com \\ Alexandre Maniçoba da Rosa Ferraz Jardim \\ ORCID: https://orcid.org/0000-0001-7094-3635 \\ Universidade Federal Rural de Pernambuco, Brasil \\ E-mail: alexandremrfj@gmail.com \\ Thieres George Freire da Silva \\ ORCID: https://orcid.org/0000-0002-8355-4935 \\ Universidade Federal Rural de Pernambuco, Brasil \\ E-mail: thigeoprofissional@hotmail.com.br
}

\begin{abstract}
Resumo
O crescimento da carcinicultura tem contribuído para diversificação alimentar, geração de empregos e renda, destacando-se assim a sua importância socioeconômica. Dentre as espécies mais cultivadas destaca-se o camarão marinho Litopenaeus vannamei, por apresentar alta resistência a diferentes condições, além de rápido crescimento e sobrevivência. Fatores ambientais como fotoperíodo podem contribuir com melhorias no sistema de produção. Este trabalho compreende uma revisão sistemática para a compreensão dos trabalhos já realizados sobre a influência do fotoperíodo em Litopenaeus vannamei. Os trabalhos foram obtidos por meio das bases: Google Scholar, Periódico da Capes e Science Direct e se referiram aos últimos quinze anos. Os termos de busca utilizados em todas as bases foram Litopenaeus vannamei and fotoperíodo e Litopenaeus vannamei and photoperiod. A busca retornou a 6.531 registros, mas apenas 5 artigos foram incluídos devidos atender os critérios de elegibilidade utilizados neste trabalho. Destes foi possível observar que $40 \%$ foram desenvolvidos em 2019. Com o resultado constatou-se que o Litopenaeus vannamei apresenta melhor desenvolvimento para alimentação, taxa de crescimento e sobrevivência, em tratamentos com exposição à luz. No entanto, esta revisão converge para o fato de que novos trabalhos devem ser desenvolvidos de modo a contemplar as relações entre a luminosidade e estágios de vida, sistemas de cultivo, parâmetros fisiológicos.
\end{abstract}

Palavras-chave: Revisão sistemática; Camarão; Bases de busca. 


\begin{abstract}
The growth of shrimp farming has contributed to food diversification, job and income generation, thus highlighting its socioeconomic importance. Among the most cultivated species, the marine shrimp Litopenaeus vannamei stands out, due to its high resistance to different conditions, in addition to fast growth and survival. Environmental factors such as photoperiod can contribute to improvements in the production system. This work comprises a systematic review to understand the work already carried out on the influence of photoperiod on Litopenaeus vannamei. The works were obtained through the bases: Scholar Google, Capes Journal and Science Direct and referred to the last fifteen years. The search terms used in all databases were Litopenaeus vannamei and photoperiod and Litopenaeus vannamei and photoperiod. The search returned 6,531 records, but only 5 articles were included due to meeting the eligibility criteria used in this work. Of these, it was possible to observe that $40 \%$ were developed in 2019 . With the result it was found that Litopenaeus vannamei presents better development for feeding, growth rate and survival, in treatments with exposure to light. However, this review converges to the fact that new works must be developed in order to contemplate the relationships between luminosity and life stages, cropping systems, physiological parameters.
\end{abstract}

Keywords: Systematic review; Shrimp; Search bases.

\title{
Resumen
}

El crecimiento de la camaronicultura ha contribuido a la diversificación alimentaria, la generación de empleo y de ingresos, destacando así su importancia socioeconómica. Entre las especies más cultivadas destaca el camarón marino Litopenaeus vannamei, por su alta resistencia a diferentes condiciones, además de rápido crecimiento y supervivencia. Los factores ambientales como el fotoperíodo pueden contribuir a mejorar el sistema de producción. Este trabajo comprende una revisión sistemática para comprender el trabajo ya realizado sobre la influencia del fotoperíodo en Litopenaeus vannamei. Los trabajos se obtuvieron a través de las bases: Scholar Google, Capes Journal y Science Direct y se refieren a los últimos quince años. Los términos de búsqueda utilizados en todas las bases de datos fueron Litopenaeus vannamei y photoperiod y Litopenaeus vannamei y photoperiod. La búsqueda arrojó 6.531 registros, pero solo se incluyeron 5 artículos debido a que cumplían con los criterios de elegibilidad utilizados en este trabajo. De estos, se pudo observar que el $40 \%$ se desarrollaron en 2019. Con el resultado se encontró que Litopenaeus vannamei presenta mejor desarrollo para la alimentación, tasa de crecimiento y supervivencia, en tratamientos con exposición a la luz. Sin embargo, esta revisión converge en que se deben desarrollar nuevos trabajos para contemplar las relaciones entre luminosidad y etapas de vida, sistemas de cultivo, parámetros fisiológicos.

Palabras clave: Revisión sistemática; Camarón; Bases de búsqueda.

\section{Introdução}

As mudanças climáticas e o aumento populacional têm provocado preocupações diversas, dentre as quais merece destaque a maximização da produção e a oferta de alimentos (Jardim et al., 2021a; Jardim et al., 2021b). Se de um lado às alterações nas condições meteorológicas do globo são evidentes (IPCC, 2014), do outro as respostas dos organismos vivos à estas condições ainda são incertas. Litopenaeus vannamei (Boone, 1931) é uma das espécies de camarão com maior destaque para a aquicultura global devido a sua resiliência e alta produção em condições ambientais diversas (i.e., salinidade, densidade, etc.) que contribuem com aspectos socioeconômicos nos locais de produção (i.e., geração de empregos e aumento da oferta de alimentos) (Maia et al., 2012; Fei et al., 2020; Rocha; Itamar, 2020). Vários estudos têm relatado as respostas deste crustáceo a condições ambientais variadas como: temperatura (Pervaiz; Sudan; Manohar, 2015), salinidade (Mendes et al., 2006), densidade (Spanghero et al., 2009), fotoperíodo (Hoang et al., 2003) e entre outros.

A luz representa um fator importante no cultivo de camarões, e seu manejo adequado contribui para melhorias no sistema de produção com consequentes reduções de custos (Coyle et al., 2011; Reis et al., 2019). O fotoperíodo pode ser definido como o tempo e horas entre o nascer e pôr do sol (Goudriaan; Van Laar, 1994). Ou ainda na concepção de Ometto (1981) este parâmetro representa o tempo em 24 horas em que é possível observar luz. Esta variável meteorológica apresenta associação com a produtividade natural, metabolismo, funcionamento do sistema imunológico, a taxa de canibalismo, a duração do ciclo, a ecdise e o desenvolvimento larval de decápodes (Ge et al., 2017). Trabalhos anteriores mostraram que a luz, também pode afetar na produção e o comportamento do camarão (Fleckenstein et al., 2019), promover melhorias na taxa de crescimento, na sobrevivência, no acúmulo de biomassa e na qualidade desse organismo (Viet et al., 2017). Além disso, observou-se incrementos na ingestão de náuplios de artêmia sob luz (Sanudin; Tuzan; Yong, 2014), bem como melhorias no ambiente de cultivo, como, 
por exemplo, melhor qualidade da água em sistema de cultivo biofloco (Reis et al., 2019). Por outro lado, mesmo havendo aumentos de produção na exposição de luz, o cultivo de camarão pode ser realizado na ausência total deste parâmetro (Baloi et al., 2013).

Os resultados observados em diferentes estudos são contraditórios e podem estar associado a fatores como espécie, estágio de crescimento, intensidade luminosa, condições ambientais, sistemas de cultivo, entre outros (Viet et al., 2017; Reis et al., 2019; Baloi et al., 2013; Fleckenstein et al., 2019). Sob esta ótica, a revisão sistemática acerca do tema pode permitir o agrupamento de informações valiosas que podem auxiliar na tomada de decisão, bem como subsidiar outros pesquisadores na realização de experimentos com base nas melhores indicações, ou mesmo servir de guia para a necessidade de novas pesquisas (Galvão; Pereira, 2014). Partindo desta perspectiva o presente trabalho busca realizar uma revisão sistemática sobre a influência do fotoperíodo no camarão (Litopenaeus vannamei Boone, 1931) e quais as principais evidências que têm sido observadas nestes estudos.

\section{Metodologia}

\subsection{Métodos}

A pergunta norteadora deste trabalho foi: “Como os trabalhos têm abordado a influência do fotoperíodo em Litopenaeus vannamei e quais as principais evidências encontradas?". A busca foi realizada nos meses de outubro e novembro de 2020, e foram obtidas informações em diferentes regiões do mundo a partir de um recorte para o período de 2005 a 2020.

\subsection{Busca e seleção dos trabalhos}

Os trabalhos foram obtidos por meio de busca nas seguintes bases: Google Scholar, Periódico da Capes e Science Direct. Os termos de busca utilizados foram: Litopenaeus vannamei and fotoperiodo e Litopenaeus vannamei and photoperiod. Posteriormente, foram observados título, resumo e palavras-chave para enquadramento do artigo dentro da temática analisada. Além disso, foram excluídos: 1) os trabalhos duplicados, 2) que não estavam publicados no idioma inglês e/ou português, 3) que não foram desenvolvidos para Litopenaeus vannamei e 4) aqueles fora do período analisado (Tabela 1).

Tabela 1. Trabalhos não incluídos devido aos critérios de elegibilidade adotados.

\begin{tabular}{lc}
\hline Critérios de Exclusão & Quantidade \\
\hline Publicados antes de 2005 & 482 \\
Outros assuntos & 3216 \\
Literatura cinzenta (teses, dissertações e resumo de eventos) & 1 \\
Repetidos & 3 \\
Idiomas que não fossem Português ou Inglês & 9 \\
Não disponíveis & 2820 \\
\hline
\end{tabular}

Elaboração da tabela: Autores.

\subsection{Análise dos dados}

Após o procedimento indicado, os trabalhos foram lidos integralmente para observância do uso de diferentes fotoperíodos, os que atenderam à esta última exigência permaneceram e foram incluídos neste estudo. Os estudos foram sintetizados e organizados em planilhas eletrônicas no Microsoft Excel ${ }^{\circledR}$ e posteriormente exportadas em tabelas; sendo obtidas 
as seguintes informações: ano de publicação, citação, referência, local, duração do estudo, base obtida, densidade de estocagem, sistema de cultivo, fotoperíodo, fase, melhor tratamento, parâmetros analisados, combinação com outros tratamentos e a revista na qual foi publicado.

\section{Resultados e Discussões}

De acordo com Cook et al. (1997) e Rother (2007) uma revisão sistemática busca reunir, avaliar e filtrar diversos trabalhos científicos desenvolvidos anteriormente e tem como finalidade responder a perguntas específicas. Essa busca pode ser realizada por meio de fontes diversas (i.e. documentos bibliográfico ou eletrônicos) a partir de critérios que permitam a obtenção de trabalhos relevantes para a temática em estudo (Rother, 2007; Galvão \& Pereira, 2014). A busca nas três bases de dados citadas anteriormente retornou 6.531 artigos (Figura 1). Para a base do Google Scholar foi possível o acesso aos 100 resultados de maior relevância ao tema. Maior parte destes foram eliminados por não apresentar conexão com o tema, 17 artigos foram lidos integralmente e, após considerados todos os critérios de elegibilidade, permaneceram nesta revisão 5 artigos (Tabela 2).

Figura 1. Fluxograma das etapas da revisão sistemática.

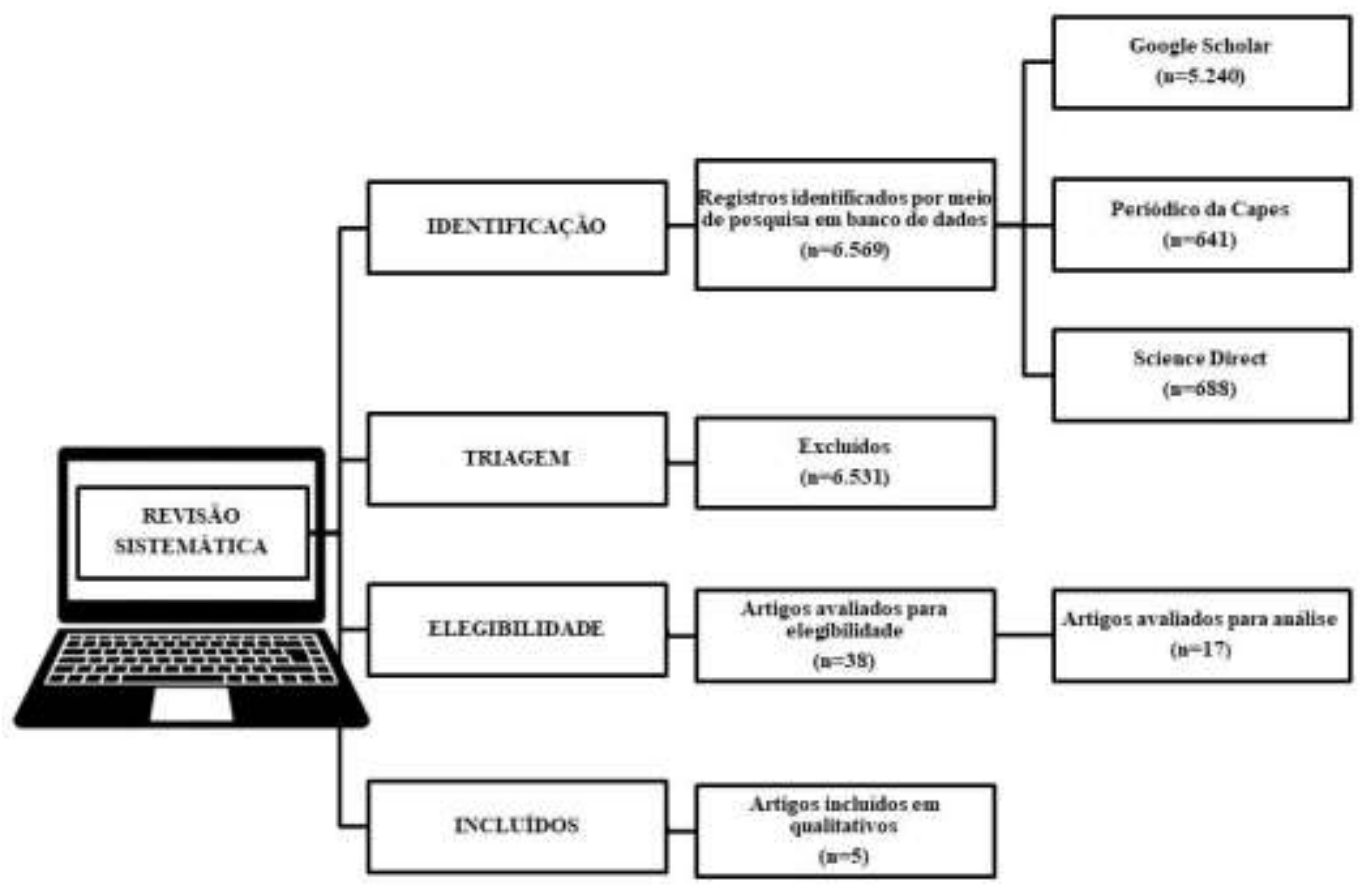

Fonte: Autores.

Cerca de 40\% dos trabalhos elegíveis foram publicados no ano de 2019 (2 estudos) os demais foram distribuídos equitativo entre os anos de 2013, 2014 e 2017 (20\% dos estudos em cada ano) (Figura 2). 
Figura 2. Relação dos trabalhos publicados por ano diferentes periódicos, durante o período de 2005-2020.

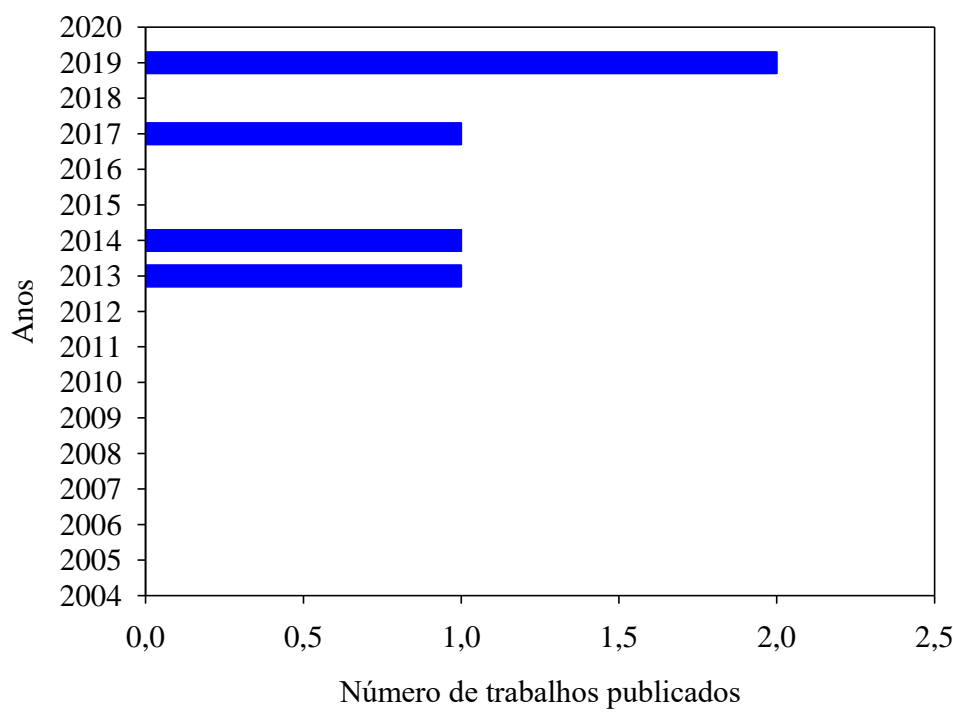

Fonte: Autores.

Durante o período observado, os trabalhos para analisar a influência do fotoperíodo em Litopenaeus vannamei foram realizados no Brasil, Malásia, Vietnã e Estados Unidos da América (Tabela 2). Experimentos realizados com fotoperíodos variados permitiram identificar qual o mais adequado para determinado cultivo. O cultivo realizado com o fotoperíodo adequado viabiliza produtividade e economia de insumos. A duração dos mesmos foi bastante variável (i.e., de 3 semanas a 90 dias) e em sua maioria ( $80 \%$ dos casos) utilizaram a combinação com o sistema de biofloco.

Tabela 2. Citações, local, duração dos experimentos, sistemas de cultivo e combinações.

\begin{tabular}{|c|c|c|c|c|c|}
\hline Citação & Título & Local & Duração & Sistema de cultivo & Tratamento \\
\hline Baloi et al. (2013) & $\begin{array}{l}\text { Performance of Pacific } \\
\text { white shrimp } \\
\text { Litopenaeus vannamei } \\
\text { raised in biofloc } \\
\text { systems with varying } \\
\text { levels of light exposure }\end{array}$ & $\begin{array}{c}\text { Florianópolis, Santa } \\
\text { Catarina, Brasil. }\end{array}$ & 40 dias & Superintensivo & Sim, Biofloco \\
\hline $\begin{array}{l}\text { Sanudin; Tuzan; } \\
\text { Yong (2014) }\end{array}$ & $\begin{array}{l}\text { Feeding Activity and } \\
\text { Growth Performance of } \\
\text { Shrimp Post Larvae } \\
\text { Litopenaeus vannamei } \\
\text { Under Light and Dark } \\
\text { Condition }\end{array}$ & $\begin{array}{c}\text { Borneo, Sabá, } \\
\text { Malásia. }\end{array}$ & 3 semanas & Sistema extensivo & Não \\
\hline Viet et al. (2017) & $\begin{array}{l}\text { Effects of photoperiods } \\
\text { on growth and quality } \\
\text { of white leg shrimp } \\
\text { (Litopenaeus } \\
\text { vannamei) in biofloc } \\
\text { system }\end{array}$ & Vietnã & 90 dias & Superintensivo & Sim, Biofloco \\
\hline
\end{tabular}




Rearing of the Pacific
white shrimp
Litopenaeus vannamei
(Boone, 1931) in BFT
system with different
photoperiods: Effects Rio Grande, RS,
on the microbial
community, water
quality and
zootechnical
performance
Effects of supplemental
LED lighting on water
quality and Pacific
white shrimp
(Litopenaeus
vannamei) performance
in intensive
recirculating

Fonte: Autores.

No sistema de bioflocos (BFT) se observa processos simbióticos que melhoram a qualidade da água, reduzem a concentração de amônia e nitrito, além de possibilitar melhorias no crescimento e resposta imunológica dos camarões (Fattah e El-Sayed, 2020). Além de controlar a comunidade microbiana no cultivo de animais, permitindo um cultivo com altas densidades de estocagem de forma sustentável, já que a troca de água é mínima (Baloi et al., 2013). De acordo com Viet et al. (2017) a intensidade de luz e o fotoperíodo também podem contribuir na formação e nos tamanhos dos flocos e na cor do camarão. Segundo Baloi et al. (2013) fotoperíodos mais longos podem resultar no crescimento de algas e da concentração de clorofila- $a$ $\left(\mathrm{C}_{55} \mathrm{H}_{72} \mathrm{O}_{5} \mathrm{~N}_{4} \mathrm{Mg}\right)$. Estes autores observaram taxas de crescimento e peso final significativamente diferente para Litopenaeus vannamei em diferentes fotoperíodos e, embora melhores resultados tenham sido obtidos com a maximização do número de horas de luminosidade, seu cultivo, em condições de escuro são aceitáveis.

Reis et al. (2019) sugerem que a presença de luz pode ter melhorado a disponibilidade de alimento natural, com reflexos positivos na taxa de crescimento relativo. Achados de Fleckenstein et al. (2019) demonstraram que os sistemas de luz total superaram os sistemas sem luz no camarão com produção em 48\% e sistemas de luz parcial em 33\%. Comprovando, que de um modo geral a associação entre aumento da exposição luminosa em consonância com o sistema BFT podem maximizar a produção de camarões, contribuindo para o aumento na oferta de alimentos.

A Tabela 3 mostra que os experimentos apresentaram a utilização de camarões em fases diferentes e variação na densidade de estocagem entre 150 indivíduos $\mathrm{m}^{-3}$ a 500 indivíduos $\mathrm{m}^{-3}$. No geral os parâmetros físico-químicos analisados foram: nitrogênio amoniacal total, nitrito, oxigênio dissolvido, temperatura, $\mathrm{pH}$, alcalinidade, salinidade, turbidez, sólidos suspensos totais e sólidos suspensos voláteis (Baloi et al., 2013; Viet et al., 2017; Reis et al., 2019). O experimento realizado por Baloi et al. (2013) não apresentou diferenças significativas entre os tratamentos nos parâmetros de temperatura, oxigênio dissolvido, pH, salinidade, amônia, nitrito e fosfato. Entretanto, os níveis médios de $\mathrm{pH}$ nos tratamentos com luz natural foram reduzidos em comparação aos experimentos com luz total e luz parcial (Fleckenstein et al., 2019). 
Estudos sobre a influência do fotoperíodo com Litopenaeus vannamei também analisaram ganho de peso, taxa de conversão alimentar, crescimento específico e sobrevivência (Sanudin et al., 2014; Viet et al., 2017; Reis et al., 2019; Fleckenstein et al., 2019). De acordo com Viet et al. (2017) o fotoperíodo 24 luz compacta 55W apresenta melhor taxa de crescimento $\left(3,69 \%\right.$ dia $\left.^{-1}\right)$, FCR $(1,68)$, taxa de sobrevivência, biomassa e qualidade (cor e tenacidade) em relação aos demais tratamentos. Em geral, os sistemas de luz total superaram os sistemas sem luz no camarão com produção de cerca de $48 \%$ e nos sistemas de luz parcial certa $33 \%$.

Tabela 3. Síntese dos achados na literatura sobre a influência do fotoperíodo em Litopenaeus vannamei. Citações, densidade de estocagem, fases dos indivíduos, fotoperíodos utilizados, parâmetros analisados e principais resultados.

\begin{tabular}{|c|c|c|c|c|c|}
\hline Citação & Densidade de estocagem & Fase & Fotoperíodos & Parâmetros & Principais resultados \\
\hline Baloi et al. (2013) & 300 indivíduos $\mathrm{m}^{-3}$ & PL10 & $\begin{array}{l}24 \mathrm{~h} \text { de luz, } 12 \mathrm{~h} \text { de luz/12h sem luz } \\
\text { e } 24 \mathrm{~h} \text { sem luz cada um com quatro } \\
\text { réplicas. }\end{array}$ & $\begin{array}{l}\text { Nitrogênio } \\
\text { amoniacal total, } \\
\text { nitrito-nitrogênio } \\
\text { e oxigênio } \\
\text { dissolvido. }\end{array}$ & $\begin{array}{l}\text { Maior produção no } \\
\text { tratamento exposto à } \\
\text { luz; mas sendo possível } \\
\text { o cultivo na ausência } \\
\text { total de luz. }\end{array}$ \\
\hline $\begin{array}{l}\text { Sanudin; Tuzan; } \\
\text { Yong (2014) }\end{array}$ & $\begin{array}{l}\text { Grupos de } 30 \text { indivíduos de } \\
\text { camarão ( } 6 \text { indivíduos } \mathrm{L}^{-1} \text { ) foram } \\
\text { introduzidos em } 7 \mathrm{~L}\end{array}$ & PL3 & $\begin{array}{l}24 \mathrm{~h} \text { de escuro, } 24 \mathrm{~h} \text { de luz e } 12 \mathrm{~h} \text { de } \\
\text { luz e } 12 \mathrm{~h} \text { de escuro }\end{array}$ & $\begin{array}{l}\text { Ganho de peso, } \\
\text { taxa de conversão } \\
\text { alimentar, } \\
\text { crescimento } \\
\text { específico } \\
\text { sobrevivência }\end{array}$ & $\begin{array}{l}\text { O camarão }(0,5 \mathrm{~cm}) \\
\text { ingeriu mais náuplios de } \\
\text { Artêmia sob a luz. Os } \\
\text { camarões TL de } 1,0 \text { e } 1,5 \\
\text { cm consumiram náuplio } \\
\text { de Artêmia igualmente } \\
\text { em ambas as condições. }\end{array}$ \\
\hline Viet et al. (2017) & 150 indivíduos $\mathrm{m}^{-3}$ & PL15 & $\begin{array}{l}24 \mathrm{~h} \text { de escuro; } 6 \mathrm{~h} \text { de luz e } 18 \mathrm{~h} \text { de } \\
\text { escuridão; } 12 \mathrm{~h} \text { de luz e } 12 \mathrm{~h} \text { de } \\
\text { escuridão; } 18 \mathrm{~h} \text { de luz e } 6 \mathrm{~h} \text { de } \\
\text { escuridão; } 24 \mathrm{~h} \text { de luz }\end{array}$ & $\begin{array}{l}\text { Taxa de } \\
\text { sobrevivência, } \\
\text { taxa de } \\
\text { crescimento, taxa } \\
\text { de crescimento } \\
\text { específico } \\
\text { produção. } \\
\text { Temperatura e pH, } \\
\text { nitrito, nitrogênio } \\
\text { amoniacal total e } \\
\text { alcalinidade. }\end{array}$ & $\begin{array}{l}\text { O fotoperíodo } 24 \mathrm{~h} \text { de luz } \\
\text { melhor taxa de } \\
\text { crescimento }\left(3,69 \% \mathrm{dia}^{-}\right. \\
\left.{ }^{1}\right), \text { FCR }(1,68) \text {, taxa de } \\
\text { sobrevivência. }\end{array}$ \\
\hline Reis et al. (2019) & 500 indivíduos $\mathrm{m}^{-3}$ & $\begin{array}{l}\text { Pós- } \\
\text { Larvas }\end{array}$ & $\begin{array}{l}\text { 1) NP - fotoperíodo natural com } \\
\text { luz natural durante o dia e coberto } \\
\text { com uma folha de plástico preta à } \\
\text { noite; 2) } 24 \mathrm{~h} \text { com luz natural } \\
\text { durante } \\
\text { dia e iluminação artificial a noite, } \\
\text { utilizando lâmpada fluorescente } \\
\text { de } 80 \text { watts; e } 3 \text { ) } 24 \mathrm{~h} \text { escuro com } \\
\text { tanques cobertos com plástico } \\
\text { preto por } 24 \text { horas. }\end{array}$ & $\begin{array}{l}\text { Desempenho } \\
\text { zootécnico, } \\
\text { temperatura, } \\
\text { oxigênio } \\
\text { dissolvido, } \\
\text { salinidade, } \\
\text { turbidez, amônia, } \\
\text { nitrito e } \\
\text { alcalinidade. }\end{array}$ & $\begin{array}{l}\text { O tratamento com } \\
\text { fotoperíodo (NP) é } \\
\text { indicado para cultura no } \\
\text { Sistema BFT. }\end{array}$ \\
\hline
\end{tabular}


Fleckenstein et al. 250 indivíduos $\mathrm{m}^{-3}$ (2019)

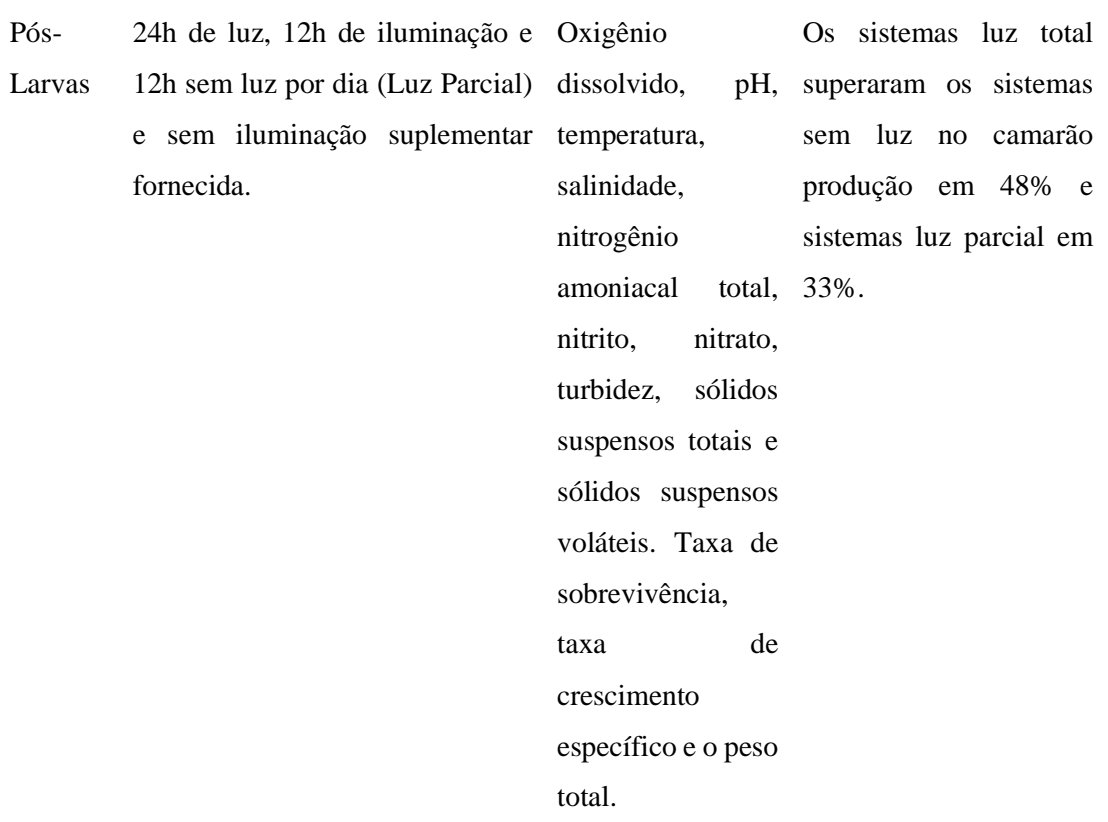

Fonte: Autores.

\section{Perspectivas}

Em cenários de escassez hídrica e necessidade de produção de alimentos para suprimento das necessidades básicas da população, a concepção de manejos sustentáveis torna-se importante. Os estudos conduzidos com Litopenaeus vannamei submetidos a diferentes fotoperíodos no período de recorte de 2005 a 2020, demonstraram a importância da variação luminosa para a criação dessa espécie. A combinação da luminosidade com a intensidade de luz em sistemas de cultivo biofloco precisam ser melhor compreendida. Não encontramos nesta revisão, trabalhos que abordem as relações entre o fotoperíodo e a qualidade dos camarões, bem como a sua relação com os aspectos ligados a locomoção destes indivíduos. Desse modo, o desenvolvimento de estudos com essa perspectiva é encorajado.

\section{Considerações Finais}

Esse estudo trouxe uma análise sistemática de trabalhos que envolvem o desempenho do camarão Litopenaeus vannamei sob a influência do fotoperíodo, mostrando que os resultados podem variar conforme o estado de crescimento, o sistema de cultivo e os fotoperíodos utilizados. Foi observado que os tratamentos com exposição à luz melhor desenvolvimento para alimentação, taxa de crescimento e sobrevivência para Litopenaeus vannamei. Contudo, os achados nessa revisão, mostraram que o cultivo sob ausência total de luz também é possível. Além disso, são necessários experimentos com outros fotoperíodos e com um intervalo de tempo maior para complementar os estudos já existentes e assim chegar a um fotoperíodo mais adequado para o cultivo.

\section{Agradecimentos}

Os autores agradecem ao Programa de Educação Tutorial (PET) da Universidade Federal Rural de Pernambuco (UFRPE) pela participação das duas primeiras autoras, e à UFRPE, pela disponibilização da infraestrutura e concessão da bolsa de Iniciação Científica da quarta autora. Também gostaríamos de agradecer a Fundação de Amparo a Ciência e Tecnologia do Estado de Pernambuco (FACEPE) e o Conselho Nacional de Desenvolvimento Científico e Tecnológico (CNPq), pela concessão das bolsas de estudo dos demais autores, e a bolsas de Produtividade em Pesquisa (PQ - 305286/2015-3) do último autor. 


\section{Referências}

Baloi, M., Arantes, R., Schveitzer, R., Magnotti, C., Binatea, L. (2013). Performance of Pacific white shrimp Litopenaeus vannamei raised in biofloc systems with varying levels of light exposure. Aquacultural Engineering, 52, 39-44.

Cook, D. J., Mulrow, C. D., Haynes, R. B. (1997). Systematic reviews: synthesis of best evidence for clinical decisions. Annals Internal Medicine, 126 (5), 376380 .

Coyle, S. D., Bright, L., A., Wood, D. R., Neal, R. S., Tidwell, J. H. (2011). Performance of Pacific white shrimp, Litopenaeus vannamei, reared in zero-exchange tank systems exposed to different light sources and intensities. Journal of the World Aquaculture Society, 42(5), 687-695.

Costa, M. S., Oliveira-Junior, J. F., Santos, P. J., Correia Filho, W. L. F., Gois, G., Blanco, C. J. C., Teodoro, P. E., Silva Junior, C. A., Santiago, D. B., Souza, E. O., Jardim, A. M. R. F. (2021). Rainfall extremes and drought in Northeast-Brazil and its relationship with El Niño-Southern Oscillation. International Journal of Climatology, 41, E2111-E2135.

Fei, F., Liu, B., Gao, X., Wang, X., Liu, Y., Bin, H. (2020). Effects of supplemental ultraviolet light on growth, oxidative stress responses, and apoptosis-related gene expression of the shrimp Litopenaeus vannamei. Aquaculture, 520, 735013.

Fleckenstein, L. J., Tierney, T. W., Fisk, J. C., Ray, A. J. (2019). Effects of supplemental LED lighting on water quality and Pacific white shrimp (Litopenaeus vannamei) performance in intensive recirculating systems. Aquaculture, 504, 219-226.

Galvão, T. F., Pereira, M. G. (2014). Revisões sistemáticas da literatura: passos para sua elaboração. Epidemiologia e Serviços de Saúde, 23(1), $183-184$.

Ge, H., Li, J., Chen, P., Chang, Z., Shen, M., Zhao, F. (2017). Cultivation of green algae Platymonas helgolandica in rearing water enhances the growth performance and resistance of Litopenaeus vannamei against Vibrio parahaemolyticus infection. Aquaculture International, 25(3), 1279-1290.

Goudriaan, J., Van Laar., H. H. (1994). Modelling potential crop growth processes: Textbook with exercices. Dordrecht, Kluwer Academic Press.

Hoang, T., Barchiesis, M., Lee, S. Y., Keenan, C. P., Marsden, G. E. (2003). Influences of light intensity and photoperiod on moulting and growth of Penaeus merguiensis cultured under laboratory conditions. Aquaculture, 216(1-4), 343-354.

IPCC. (2014). Contribuição do Grupo de Trabalho II para o Quinto Relatório de Avaliação do Painel Intergovernamental sobre Alterações Climáticas (IPCC). [s.l: s.n.].

Jardim, A. M. R. F., Silva, T. G. F., Souza, L. S. B., Araújo Júnior, G. do N., Alves, H. K. M. N., Souza, M. S., Araújo, G. G. L., Moura, M. S. B. (2021a). Intercropping forage cactus and sorghum in a semi-arid environment improves biological efficiency and competitive ability through interspecific complementarity. Journal of Arid Environments, 188, 104464.

Jardim, A. M. R. F., Souza, L. S. B., Alves, C. P., Araújo, J. F. N., Souza, C. A. A., Pinheiro, A. G., Araújo, G. G. L., Campos, F. S., Tabosa, J. N., Silva, T. G. F. (2021b). Intercropping forage cactus with sorghum affects the morphophysiology and phenology of forage cactus. African Journal of Range and Forage Science, 38:1-12.

Maia, E. de P., Modesto, G. A., Brito, L. O., Gálvez, A. O. (2012). Crescimento, sobrevivência e produção de Litopenaeus vannamei cultivado em sistema intensivo. Pesquisa Agropecuária Pernambucana, 17(1), 15-19.

Mendes, P. D. P., Albuquerque, M. L. L. T., Queiroz, D. M., Lima, A. C., Lopes, Y. V. A. (2006). Aclimatação do camarão marinho <em>Litopenaeus vannamei</em> (Boone, 1931) à água doce com diferentes estratégias de alimentação e calagem. Acta Scientiarum. Animal Sciences, 28(1), 89-95.

Ometto, J. C. (1981). Bioclimatologia vegetal. São Paulo: Agronômica Ceres.

Pervaiz, P. A., Sudan, M., Manohar, S. (2015). Studies on the effect of photoperiodism and temperature on moulting of a freshwater prawn Macrobrachium dayanum. International Journal of Fisheries and Aquatic Studies, 3(1), 325-328.

Pontes, C. S. (2006) Padrão de deslocamento do camarão marinho Litopenaeus vannamei (Boone) (Crustacea, Decapoda, Penaeidae) nas fases clara e escura ao longo de 24 horas. Revista Brasileira de Zoologia, 23(1), 223-227.

Reis, W. G., Wasielesky-Junior, W., Abreu, P. C., Brandão, H., Krummenauer, D. (2019). Rearing of the Pacific white shrimp Litopenaeus vannamei (Boone, 1931) in BFT system with different photoperiods: Effects on the microbial community, water quality and zootechnical performance. Aquaculture, 508,19-29.

Rocha, I. Camarão Marinho Cultivado. Associação Brasileira de Criadores de Camarões (ABCC). Disponível em: https://abccam.com.br/ -Acessado em 03 de dezembro de 2020.

Rother, E. T. (2007). Revisão sistemática X revisão narrativa. Acta Paul Enferm, 20(2), v-vi.

Sanudin, N., Tuzan, A. D., Yong, A. S. K. (2014). Feeding Activity and Growth Performance of Shrimp Post Larvae Litopenaeus vannamei Under Light and Dark Condition. Journal of Agricultural Science, 6(11), 103-109.

Siddhartha Pati, A. C., Dash, B. P. (2015). A Study on the Growth of Juveniles of Tiger Prawn, Penaeus monodon (Fabricius) Under Different Photoperiods. Journal of Aquaculture Research \& Development, 6 (12), 1000385.

Spanghero, D. B. N., Silva, U. L., Pessoa, M. N. C., Mederos, E. C. A. M., Oliveira, I. R., Mendes, P. P. (2009). Utilização de modelos estatísticos para avaliar dados de produção do camarão <em>Litopenaeus vannamei</em> cultivados em águas oligohalina e salgada. Acta Scientiarum. Animal Sciences, 30(4), 451458 .

Viet, L. Q., Hai, T. N., Minh, P. T. (2017). Effects of photoperiods on growth and quality of white leg shrimp (Litopenaeus van-namei) in biofloc system. Can Tho University Journal of Science, 06, 83-92. 